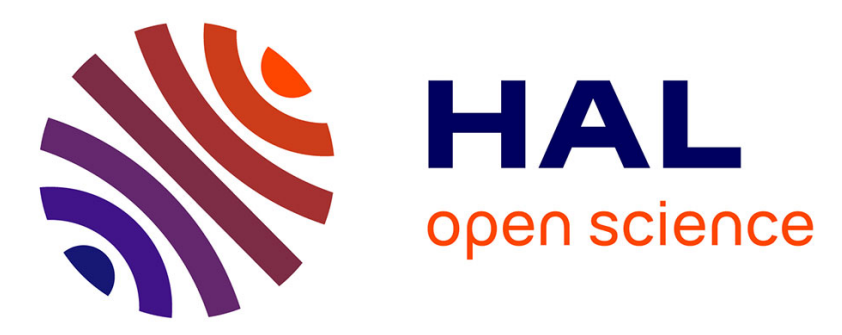

\title{
Correlation of the soft modes in the orthorhombic and cubic phases of $\mathrm{KNbO} 3$
}

\author{
A.M. Quittet, J.L. Servoin, François Gervais
}

\section{To cite this version:}

A.M. Quittet, J.L. Servoin, François Gervais. Correlation of the soft modes in the orthorhombic and cubic phases of KNbO3. Journal de Physique, 1981, 42 (3), pp.493-498. 10.1051/jphys:01981004203049300 . jpa-00209034

\section{HAL Id: jpa-00209034 https://hal.science/jpa-00209034}

Submitted on 1 Jan 1981

HAL is a multi-disciplinary open access archive for the deposit and dissemination of scientific research documents, whether they are published or not. The documents may come from teaching and research institutions in France or abroad, or from public or private research centers.
L'archive ouverte pluridisciplinaire HAL, est destinée au dépôt et à la diffusion de documents scientifiques de niveau recherche, publiés ou non, émanant des établissements d'enseignement et de recherche français ou étrangers, des laboratoires publics ou privés. 


\title{
Correlation of the soft modes in the orthorhombic and cubic phases of $\mathrm{KNbO}_{3}$
}

\author{
A. M. Quittet \\ Laboratoire de Physique des Solides (*) Université Paris-Sud, Bâtiment 510, 91405 Orsay, France \\ J. L. Servoin and F. Gervais \\ Centre de Recherches sur la Physique des Hautes Températures, C.N.R.S., 45045 Orléans, France \\ (Reçu le 12 février 1980, révisé le 6 novembre, accepté le 1er décembre 1980)
}

\begin{abstract}
Résumé. - Nous présentons les résultats de mesures de réflectivité infrarouge dans la phase cubique de $\mathrm{KNbO}_{3}$, ce qui permet d'atteindre les modes polaires dans cette phase dans laquelle il n'existait aucune donnée jusqu'à maintenant. Lorsqu'on compare avec des mesures par diffusion Raman, on constate que la fréquence du " mode mou " varie de façon à peu près continue de la phase cubique (mode $F_{1 u}$ ) à la phase orthorhombique (mode $B_{2}$ ), malgré les transitions du premier ordre qui les séparent. Chaque transition correspond à la stabilisation d'une composante du mode mou lorsque la température décroît.

Nos résultats confirment que les transitions de phase dans ce matériau ont un caractère displacif prépondérant aux températures très supérieures à la transition ferro-paraélectrique, tandis que, près de cette transition et en dessous, l'anharmonicité croissante modifie le comportement qui, de displacif, devient celui d'un désordre dynamique.
\end{abstract}

\begin{abstract}
We have made the first measurements of the polar modes in the cubic phase of the ferroelectric perovskite $\mathrm{KNbO}_{3}$ by infrared reflectivity studies. Comparison of these frequencies with those measured by Raman scattering shows that the "soft mode " frequency varies almost continuously from the three-dimensional $F_{1 u}$ mode in the cubic phase to the one-dimensional $B_{2}$ mode in the orthorhombic phase, in spite of the first order transitions separating these phases. Each transition corresponds to the stabilization of one component of the soft mode on cooling.

These data confirm that the phase transitions have a mainly displacive character well above the ferro-paraelectric transition while near and below this transition, the increasing anharmonicity gives rise to modification of the displacive behaviour becoming dynamically disordered.
\end{abstract}

1. Introduction. - $\mathrm{KNbO}_{3}$ has three ferroelectric phases at temperatures below $698 \mathrm{~K}$ and one paraelectric phase above $698 \mathrm{~K}$ (see Fig. 4).

The polar modes of vibration have been extensively studied since they can be correlated with the dielectric properties and therefore with the nature of the phase transitions. In the ferroelectric phases, Raman scattering studies have most frequently been made [1-5] and in a few cases the results of these studies have been compared to infrared reflectivity measurements [6].

However, in the cubic phase, only the infrared experiments can be performed, since, the phase being

(*) Associé au C.N.R.S. centrosymmetric, the polar modes are Raman inactive. We present the first infrared reflectivity results obtained in the cubic phase and correlate these with measurements by Raman scattering.

2. Cubic phase : infrared reflectivity. -2.1 SYMMETRY GROUP ANALYSIS. - The irreducible representations of the normal modes in cubic $\mathrm{KNbO}_{3}$ (point group, $\mathrm{O}_{\mathrm{h}}$ ) are as follows :

- $1 \mathrm{~F}_{2 \mathrm{u}}$ mode, Raman and infrared inactive;

- $3 \mathrm{~F}_{1 \mathrm{u}}$ polar optic modes, infrared active only;

$-1 \mathrm{~F}_{1 \mathrm{u}}$ acoustic mode.

All these modes are threefold degenerate, so that the infrared reflectivity which is directly related to the 
polar modes will be independent of the polarization of the light beam.

2.2 EXPERIMENTAL DEVICE. - The infrared reflectivity spectra have been obtained with the aid of a BRUKER IFS 113C scanning interferometer. The interferogram is analysed by an on-line computer : the reflectivity spectrum is thus obtained immediately. The spectrometer has been completed with a high temperature equipment which allows measurements to be performed up to $1400 \mathrm{~K}$. Problems associated with the thermal emission of the sample itself have been solved, as described in an earlier paper [7].

2.3 EXPERIMENTAL RESULTS AND ANALYSIS. - A spectrum measured at $740 \mathrm{~K}, 40 \mathrm{~K}$ above the ferroparaelectric transition is shown as open circles in figure 1. It has been analysed by both Kramers-Krönig inversion and the factorized model of the dielectric function $[8,9]$. In the tetragonal ferroelectric phase

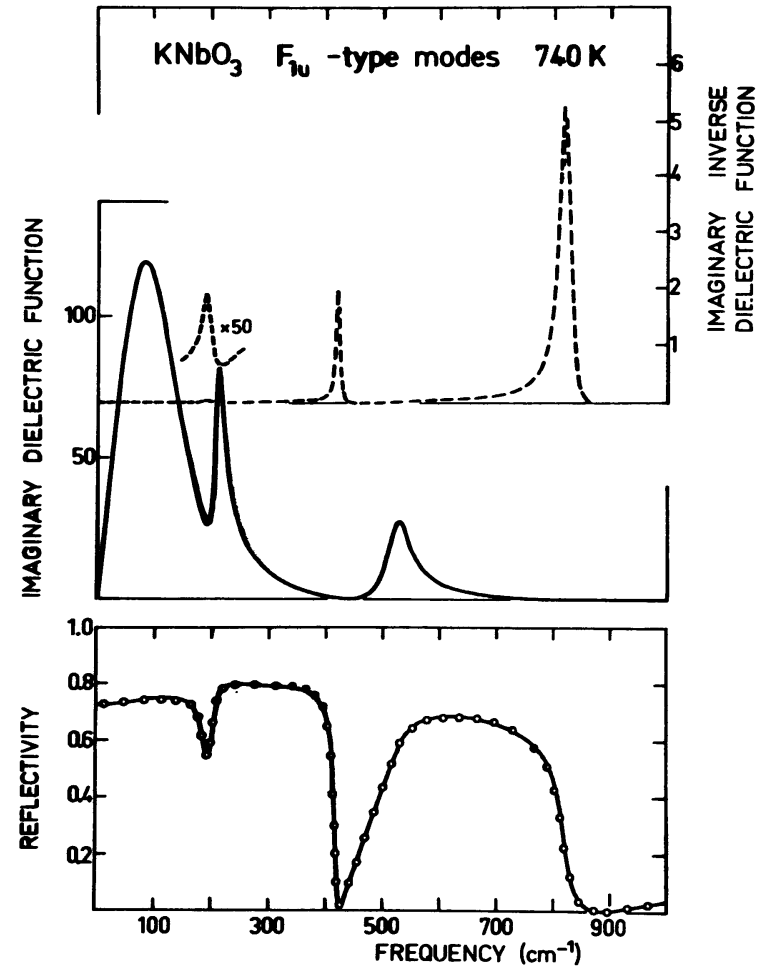

Fig. 1. - Infrared reflection spectrum in cubic $\mathrm{KNbO}_{3}$ at $740 \mathrm{~K}$. Open circles are experimental data. The full line is a fit to equation (1) with the parameters listed in table I. The imaginary parts of the dielectric and inverse dielectric functions calculated with equation (1) correspond to the structure of TO and LO modes, respectively. of $\mathrm{BaTiO}_{3}$, this model is in excellent agreement with Raman measurements [10], whereas the classical dispersion theory fails to describe wide reflection bands. In this model, the dielectric function is given by :

$$
\varepsilon=\varepsilon_{\infty} \prod_{j} \frac{\Omega_{j \mathrm{LO}}^{2}-\omega^{2}+i \gamma_{j \mathrm{LO}} \omega}{\Omega_{j \mathrm{TO}}^{2}-\omega^{2}+i \gamma_{j \mathrm{TO}} \omega}
$$

where the frequencies and dampings of the transverse polar optic modes and their corresponding longitudinal mode are ajustable parameters. $\varepsilon_{\infty}$ is the optical high frequency dielectric constant.

The four parameters corresponding to each pair of TO-LO modes are found by fitting equation (1) to the experimental data. The fit is shown in figure 1 as the full line through the experimental points and the values of the parameters are given in table I with $\varepsilon_{\infty}=5.55$

In this model, each oscillator strength can. be calculated from

$$
\Delta \varepsilon_{j}=\varepsilon_{\infty} \frac{\Omega_{j \mathrm{LO}}^{2}-\Omega_{j \mathrm{TO}}^{2}}{\Omega_{j \mathrm{TO}}^{2}} \prod_{k \neq j} \frac{\Omega_{k \mathrm{jO}}^{2}-\Omega_{j \mathrm{TO}}^{2}}{\Omega_{k \mathrm{TO}}^{2}-\Omega_{j \mathrm{TO}}^{2}} .
$$

Note that the oscillator strength in the classical model of independent oscillators is the product of the first two terms of the right hand term. When $\omega=0$, equation (1) leads to the Lyddane-Sachs-Teller (L.S. T.) relation giving the static dielectric constant as,

$$
\varepsilon(0)=\varepsilon_{\infty} \prod_{j}\left(\frac{\Omega_{j \mathrm{LO}}}{\Omega_{j \mathrm{TO}}}\right)^{2} .
$$

With the values given in table $\mathrm{I}$, one obtains $\varepsilon_{0}=159$ (which can also be self consistently calculated from $\left.\varepsilon_{\infty}+\sum_{j} \Delta \varepsilon_{j}\right)$

Although the lowest frequency transverse mode turns out to be at the limit of the overdamping criterion $\gamma / \Omega \simeq \sqrt{2}$ its frequency can easily be determined from an infrared reflectivity experiment. All the other transverse and longitudinal modes are sufficiently underdamped that their frequencies can be accurately determined. The low frequency reflectivity $R_{0}$ gives directly the frequency $\Omega_{\text {od }}$ of the (over-)damped mode from equation (1) and the L. S. T. relation,

$$
\Omega_{\mathrm{od}}=\sqrt{\varepsilon_{\infty}} \frac{1-\sqrt{R_{0}}}{1+\sqrt{R_{0}}} \prod_{j} \Omega_{j \mathrm{LO}} / \prod_{j \neq \mathrm{od}} \Omega_{j \mathrm{TO}} .
$$

Table I.

\begin{tabular}{ccccccc}
$\Omega_{j \mathrm{TO}} \mathrm{cm}^{-1}$ & $\Omega_{j \mathrm{LO}} \mathrm{cm}^{-1}$ & $\gamma_{j \mathrm{TO}} \mathrm{cm}^{-1}$ & $\gamma_{j \mathrm{LO}} \mathrm{cm}^{-1}$ & \multicolumn{2}{c}{$\Delta \varepsilon_{j}$} & \multicolumn{2}{c}{ oscil. str. } & TO & LO \\
-115 & - & - & - & - & - & - \\
207 & 420 & 165 & 7 & 141 & 57 & 425 \\
522 & 193 & 19 & 19 & 9 & 196 & 188 \\
& 820 & 54 & 22 & 3 & 513 & 830
\end{tabular}


This method of determination of the frequency of this important mode can be used in circumstances where the experimental conditions are not adequate to determine all the frequencies. It turns out that only this low frequency mode varies significantly with temperature (see Table I), so that the low frequency reflectivity displays the variation of this mode since $\Omega_{\text {od }}$ is a monotonically decreasing homographic function of $\sqrt{R_{0}}$.

The correlation of this low frequency mode (L.F. mode) with the low frequency $\mathrm{B}_{2}(\mathrm{TO})$ underdamped mode observed in the orthorhombic phase by Raman scattering is discussed below.

3. Orthorhombic phase : Raman scattering. -3.1 SYMMETRY GROUP ANALYSIS. - The unit cell of the orthorhombic phase is simply the cubic cell distorted, but with two of its axes along the diagonals of a face of the cubic cell (the ferroelectric $X$ axis and $Y$ axis) and the third axis parallel to the perpendicular edge. The space group is $\mathrm{C}_{2 \mathrm{~V}}^{14}$ and the normal modes are to be determined in the $\mathrm{C}_{2 \mathrm{v}}$ point group. With one molecular unit $\mathrm{KNbO}_{3}$ per cell, there are 12 optic modes :

$-4 A_{1}$ polar optic modes. Dipolar momentum parallel to $X$

- $4 B_{1}$ polar optic modes. Dipolar momentum parallel to $Y$

$-3 \mathrm{~B}_{2}$ polar optic modes. Dipolar momentum

$-1 \mathrm{~A}_{2}$ non polar optic mode. parallel to $Z$

Though the $\mathrm{C}_{3 \mathrm{v}}$ symmetry group of the rhombohedral phase is not a subgroup of the $\mathrm{C}_{2 \mathrm{v}}$ group of the orthorhombic phase and though the transition between these phases is of first order, a mode can be related to the transition if it is at least partially of displacive type : such a mode must belong to the $B_{2}$ irreducible representation since only in this case can the dipolar momentum rotate the spontaneous polarization in the correct plane of the unit cell to be compatible with the rhombohedral configuration.

$3.2 \mathrm{~B}_{2}(\mathrm{TO})$ FERROELECTRIC MODE, AND ITS TEMPERATURE DEPENDENCE. - All the normal optic modes have been observed in their transverse configuration at room temperature $[2,5]$. The lowest frequency mode of the $B_{2}$ irreducible representation has been studied as a function of temperature in the whole of the orthorhombic phase (Fig. 2) : since the temperature has a noticeable effect only on this mode, it is presumed to be the ferroelectric mode.

Since this mode is highly damped, its frequency can only be determined by fitting a model to the experimental data. We found that the classical damped oscillator model was adequate, which means the scattering response is proportional to

$$
(n+1) \operatorname{Im} \frac{1}{\omega^{2}-\omega_{0}^{2}+i \gamma \omega}
$$

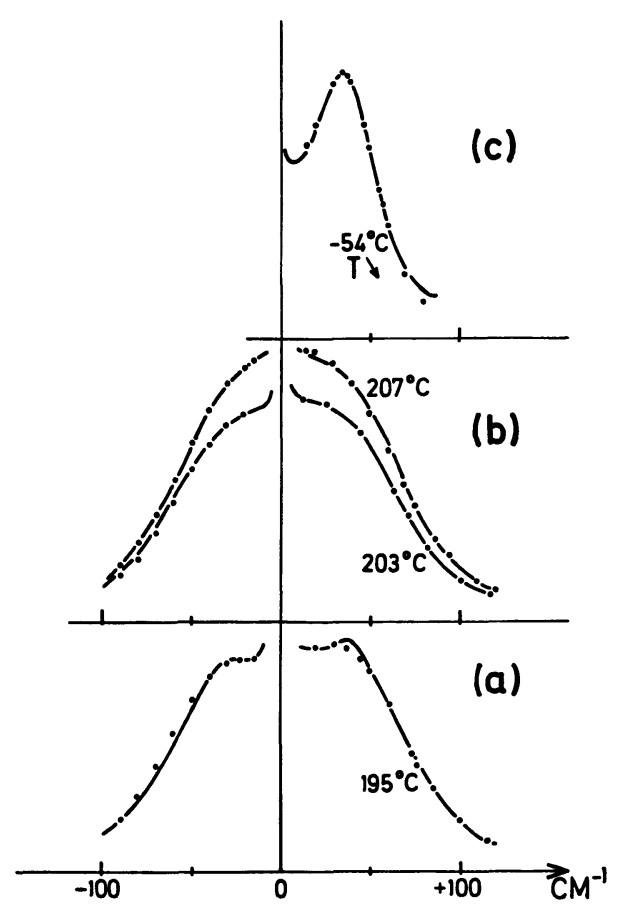

Fig. 2. - Raman spectra of the $B_{2}(\mathrm{TO})$ soft mode at different temperatures in the orthorhombic phase of $\mathrm{KNbO}_{3}$. Full line : experimental spectrum. Dots : calculated points.

where $n$ is the mean number of phonons :

$$
\frac{1}{\exp \frac{\hbar \omega}{k_{\mathrm{B}} T}-1}
$$

$\omega_{0}$ and $\gamma$ are shown in figure 3 as a function of temperature. The frequency $\omega_{0}$ decreases smoothly on cooling, without any critical behaviour, whereas the damping constant diverges slightly near the orthorhombic tetragonal transition [12]. Thus, this mode becomes slightly overdamped near the transition which it is not related to but it disappears at the rhombohedral-orthorhombic transition $[12,13]$.

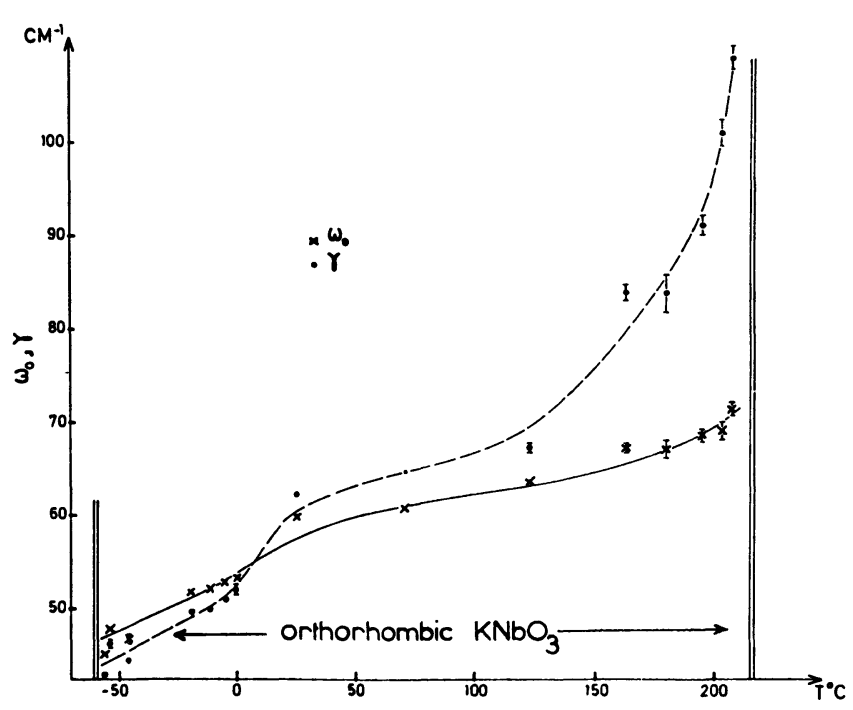

Fig. 3. - Frequency, $\omega_{0}$, and damping, $\gamma$, of the $\mathbf{B}_{2}$ (TO) soft mode in the orthorhombic phase of $\mathrm{KNbO}_{3}$ as a function of temperature. 
4. Discussion. - 4.1 ConCERning the teTragoNAL PHASE. - Precise measurements could not be made in the tetragonal phase because it was not possible, with our experimental set up to obtain or maintain a single domain. However, noting the comment at the end of $\S 2.3$, continuous monitoring of the low frequency reflectivity through the tetragonal phase during heating, shows that a very damped (may be slightly overdamped) mode exists in this phase, whose frequency monotonically increases but lies between those of the L.F. modes in the orthorhombic and cubic phases.

Recently, M. D. Fontana et al. [16] carried out an inelastic neutron scattering study on a single domain sample of $\mathrm{KNbO}_{3}$ in the tetragonal phase at $T=518 \mathrm{~K}$. Fontana et al. find a highly damped mode at the Brillouin zone centre, with a frequency of $1.6 \pm 0.5 \mathrm{THz}$ (or $55 \pm 15 \mathrm{~cm}^{-1}$ ) which they call the "Ferroelectric Mode». There is a large uncertainty in the frequency because the neutron groups are not very well defined and the authors do not fit a theoretical model of a damped mode to their experimental data it is well known that the real frequency is always higher than the frequency of the maximum at the neutron group.

4.2 CORRELATION OF THE SOFT MODES IN ALL PHASES AND COMPARISON WITH $\mathrm{BaTiO}_{3}$. - The frequency of the L.F. mode observed at $740 \mathrm{~K}$ in the cubic phase sits on the curve obtained by extrapolating the low frequency $\mathrm{B}_{2}(\mathrm{TO})$ mode curve in the orthorhombic phase, and the interpolated frequency in the tetragonal phase is compatible with the results described in the above paragraph. This observation will be compared with what has been obtained on the isomorphous compound $\mathrm{BaTiO}_{3}$.

$\mathrm{KNbO}_{3}$ and $\mathrm{BaTiO}_{3}$ display exactly the same sequence of phases with temperature, although the transition temperatures are different. The diffuse $\mathrm{X}$-ray scattering patterns [17] are similar in all phases as is the anomalous Raman spectrum in the cubic phase [18, 19]. However $\mathrm{BaTiO}_{3}$ has been more studied in its tetragonal phase as it is easier to obtain this compound as a single domain in this phase. The " continuity " of the frequency of the «ferroelectric mode " from the tetragonal [20] to the cubic [21] phase has been demonstrated in $\mathrm{BaTiO}_{3}$ by Raman scattering and infrared reflectivity studies, as shown in figure 4. By comparing the behaviour of both materials, we have shown that in $\mathrm{KNbO}_{3}$ there is a mode whose frequency decreases continuously from the cubic to the orthorhombic phases but with some additional effects near the transitions. In the rhombohedral phase, this mode has a much higher frequency $[12,13]$.

This result is strongly related to the succession of phases which characterizes $\mathrm{BaTiO}_{3}$ and $\mathrm{KNbO}_{3}$. In the paraelectric cubic phase, the ferroelectric mode is 3-fold degenerate $\left(F_{1 u}\right)$. At each successive transition one component only becomes stable, the other component(s) remaining soft (E-type in the tetragonal

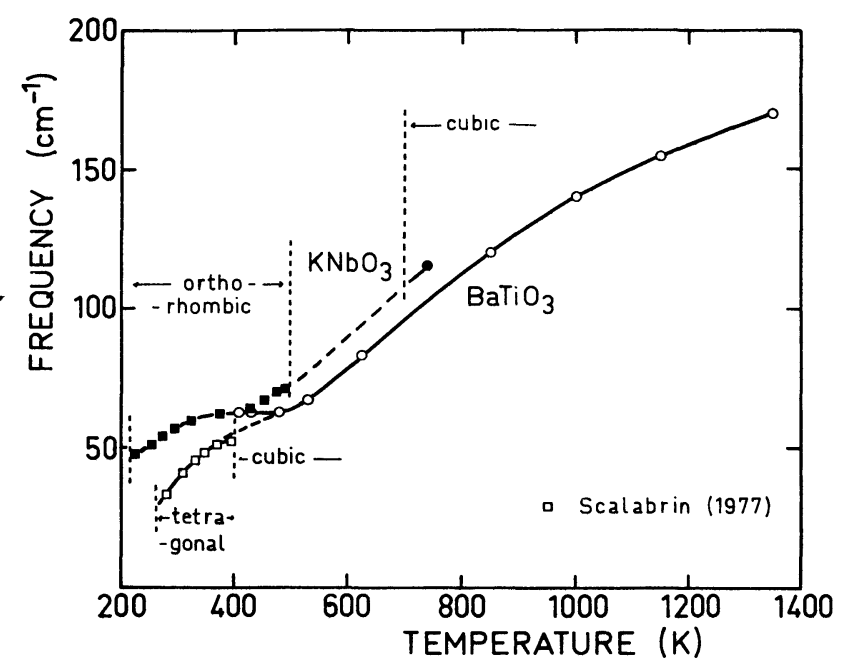

Fig. 4. - Comparison of the frequencies of the modes in the cubic phase $\left(\mathrm{O} \mathrm{BaTiO}_{3}[16],-\mathrm{KNbO}_{3}\right)$ with the frequency ( $\square$ ) of the $\mathrm{B}_{2}$ soft mode in orthorhombic $\mathrm{KNbO}_{3}$ and with the $\mathrm{E}$ soft mode $(\square)$ in tetragonal $\mathrm{BaTiO}_{3}[15]$.

phase, $\mathbf{B}_{2}$-type in the orthorhombic phase) so that there is no soft mode left in the lowest temperature phase (rhombohedral). However, there is no direct correlation between the modes in one phase and those of the lower temperature phase since the transitions are all of first order and the symmetry groups are not successive subgroups of that of the paraelectric phase, except for the tetragonal phase. For example, one component of the "ferroelectric " $E$ mode of the tetragonal phase yields the low frequency $B_{2}$ mode of the orthorhombic phase (since its polarization is parallel to the preserved cubic axis) while the other component must be combined with a $\mathrm{A}_{1}$ mode of the tetragonal phase to give a combination of one $A_{1}$ and one $B_{1}$ normal modes of the orthorhombic phase.

The behaviour described above is characteristic of the successive transitions. In contrast one may observe that in $\mathrm{PbTiO}_{3}$ which has only the high temperature-transition, the $F_{1 u}$ soft mode of the cubic phase splits into one $A_{1}+$ one $E$ mode in the tetragonal phase, whose frequencies increase on cooling [22] while their damping constants decrease.

4.3 AbOUt THE MECHANISM OF THE TRANSITIONS IN $\mathrm{KNbO}_{3}$. - 4.3.1 Static dielectric constant. The existence of a L.F. polar mode whose frequency decreases through the transitions may be related to a displacive character of the transitions. Such a character can be examined by comparing the experimental static dielectric constant to that calculated by the L. S. T. relation from the frequencies of the polar modes. In the orthorhombic phase at $300 \mathrm{~K}$, the experimental and calculated dielectric constants are approximately equal in the direction of the three orthorhombic axes (calculated : Refs [2, 4, 12]; measured : Ref. [14]).

The measurements of the $B_{2}$ soft mode as a function of temperature allow us to compare the calculated and the measured values [14] of the dielectric constant 
Table II.

\begin{tabular}{rlcl}
\multicolumn{1}{l}{$T$} & $\omega_{0(T)}$ & $\varepsilon$ calc. & $\varepsilon$ exper. \\
$-\quad 20$ & 51 & - & - \\
0 & 54 & 912 & 900 \\
+20 & 57 & 730 & 820 \\
50 & 60 & 659 & $715 \pm 50$ \\
100 & 62 & 617 & 650 \\
150 & 64 & 580 & 600 \\
180 & 67 & 528 & 600 \\
200 & 69.5 & 490 & 600 \\
215 & 71 & 470 & $610 \pm 40$
\end{tabular}

Observations

The calculated value has been obtained from the complete L. S. T. relation using measured values of the TO and LO $\mathrm{B}_{2}$ modes by Raman scattering spectroscopy $[2,4,12]$.

The two experimental values given with an uncertainty are taken from table III of E. Wiesendarger's paper [14], the other values are extrapolated from figure 1 in the same paper.

in the $Z$ direction in the whole of the orthorhombic phase. The L.F. mode is the only $B_{2}$ mode which has a significant temperature dependence (the frequency of the other $B_{2}$ modes is invariant to within $0.5 \%$ ) so that the static dielectric constant calculated from the L.S. T. relation reduces to :

$$
\varepsilon(T)=\varepsilon(20) \times\left(\frac{\omega_{0(20)}}{\omega_{0(T)}}\right)^{2}
$$

where $\omega_{0(20)}$ and $\omega_{0(T)}$ are the frequencies of the soft mode at $20^{\circ} \mathrm{C}$ and $T{ }^{\circ} \mathrm{C}$ respectively. The values are given in table II.

The calculated and measured values are in good agreement at the lower temperatures, but there is an increasing discrepancy for temperatures higher than $150^{\circ} \mathrm{C}$. This suggests that there are two contributions to the dielectric constant, one from the polar phonons, and one from other excitations or relaxations.

In the cubic phase, there is a huge discrepancy between the value 159 of the dielectric constant calculated in $\S 2$ by the L. S. T. relation from the infrared reflectivity and the measured value, 2800 , at the same temperature $[14,15]$.

4.3.2 Displacive and/or order-disorder character of the transitions. - The strong displacive character has been demonstrated in the orthorhombic phase because the dielectric constant due to polar módes calculated from the L. S. T. relation accounts for the measured clamped dielectric constant, except near the orthorhombic-tetragonal transition (Table II).

At $40 \mathrm{~K}$ above the ferro-paraelectric transition where the frequencies of the polar modes have been obtained, the calculated dielectric constant due to the polar modes (L. S. T. relation) accounts for only a small part of the measured dielectric constant. The same phenomenon is seen in $\mathrm{BaTiO}_{3}[21]:$ in the latter material, it has been shown that the discrepancy decreases rapidly when the temperature is increased. These observations show that, although the behaviour of these crystals far from the transitions is that of a standard displacive type, within a range of temperature encompassing the two high-temperature phase transitions, this behaviour is dominated by another mechanism which leads to the first order character of the transitions. A pure displacive mechanism should lead to a second order transition.

S. Aubry [26] and T. Schneider et al. [27] have constructed models which describe displacive, orderdisorder and any mixed type of structural transition. They show that there can be a large variety of scattering spectra. When the displacive aspect dominates, the quasiharmonic modes show up as well defined peaks that could be termed underdamped phonons. The results presented in this paper show that one mode is no longer quasiharmonic when it becomes overdamped. Moreover, in the case of $\mathrm{BaTiO}_{3}$ the overdamping is such that one cannot distinguish the peak centred at zero frequency and which is nearly Lorentzian and a pure Lorentzian peak which would be obtained from a relaxation.

In conclusion, it appears that the transition in $\mathrm{KNbO}_{3}$ is mainly of displacive character but with a large anharmonicity which reflects a tendency of the potential well to be flatter than an harmonic one and which may indicate a multiple well with low barriers in the cubic and tetragonal phases. In the orthorhombic phase the displacive aspect is stronger.

Acknowledgments. - The authors are indebted to U. Fluckiger who provided the excellent $\mathrm{KNbO}_{3}$ sample and to Prof. M. Lambert and Drs. R. Comes and Y. Luspin for valuable discussions.

\section{References}

[1] Perry, C. H., TORNERg, N. E., Light scattering spectra of solids (Springer-Verlag, Berlin-Heidelberg-New York) 1969 , p. 467.

[2] Winter, F. X., Wiesendanger, E., Claus, R., Phys. Status Solidi (b) 64 (1974) 95. Phys. Status Solidi (b) 72 (1975) 189.
[3] Fukumoto, T., OKamoto, A., Hattori, T., Mitsuishi, A., Solid State Commun. 17 (1975) 427.

[4] Hurrel, J. P., Bozinis, D. G., Solid State Commun. 17 (1975) 1541 . 
[5] Quittet, A. M., Bell, M. I., Krauzman, M., Raccah, P. M., Phys. Rev. B 14 (1976) 5068.

[6] Bozinis, D. G., Hurrel, J. P., Phys. Rev. B 13 (1976) 3109.

[7] Gervais, F., Servoin, J. L., Infrared Phys. 18 (1978) 883.

[8] Berreman, D. G., Unterwald, F. C., Phys. Rev. 174 (1968) 791.

[9] Gervais, F., Piriou, B., J. Phys. C 7 (1974) 2374 Gervais, F., Serrvoin, J. L., Appl. Opt. 16 (1977) 2952.

[10] Servoin, J. L., Gervais, F., Quittet, A. M., Luspin, Y., Phys. Rev. B 21 (1 March 1980) 2038.

[11] Gervais, F., Luspin, Y., Servoin, J. L., Quittet, A. M., 4th EMF-Portoroz (3-7 sept. 1979), to be published in Ferroelectrics.

[12] QuitteT, A. M., Thesis, Université Paris-Sud, Orsay-no 1991 (1978).

[13] Fontana, M. P., Razzeti, C., Solid State Commun. 17 (1975) 377.

[14] Wiesendager, E., Ferroelectrics 6 (1974) 263.

[15] Triebwasser, S., Phys. Rev. 101 (1956) 993.

[16] Fontana, M. D., Dolling, G., Kugel, G. E., CarrabaTos, C., Phys. Rev. B 20 (1979) 3850.

[17] Comes, R., LAmbert, M. and GuINIER, A., Solid State Commun. 6 (1968) 715 and J. Phys. Soc. Japan 28 Suppl. 195 (1970).
[18] QuitteT, A. M., Lamber T, M., Solid State Commun. 12 (1973) 1053 for $\mathrm{BaTiO}_{3}$.

[19] Quittet, A. M., Fontana, M., Lambert, M., WiesendanGER, E., Ferroelectrics 8 (1974) 858 for $\mathrm{KNbO}_{3}$.

[20] Scalabrin, A., Chaves, A. S., Shim, D. S. and Porto, S. P. S., Phys. Status Solidi (b) 79 (1977) 739.

[21] Luspin, Y., Servoin, J. L., Gervais, F., Proc. of the 4th European Conference on Ferroelectricity, Portoroz (1979), Ferroelectrics (in press).

Luspin, Y., Servoin, J. L. and Gervais, F., J. Phys. C. Solid State Phys. 13 (1980) 3761.

[22] Burns, G., Scott, B. A., Phys. Rev. B 7 (1973) 3088.

[23] Currat, R., Comes, R., Dorner, B., Wiesendanger, E., $J$. Phys. C. Solid State Physics 7 (1974) 2521.

[24] Perry, C. H., Buhay, H., Quittet, A. M., Currat, R., in Lattice Dynamics, edited by M. Balkanski (Flammarion, Paris) 1978, p. 677.

[25] Comes, R., Shirane, G., Phys. Rev. B 5 (1972) 1886.

[26] Aubry, S., J. Chem. Phys. 62 (1975) 3217 and J. Chem. Phys. 64 (1976) 3392.

[27] Schneider, T. and Stoll, E., Phys. Rev. B 10 (1974) 2004 and Phys. Rev. B 13 (1976) 1216. 